\title{
Core Decompression and Cancellous Bone Grafting for Avascular Necrosis of the Femoral Head - Short Term Follow Up
}

\author{
Debojyoti Mukherjee ${ }^{1}$, Ranadip Halder² \\ ${ }^{1,2}$ Department of Orthopaedics, R G Kar Medical College, Kolkata, West Bengal, India.
}

\section{ABSTRACT}

\section{BACKGROUND}

Core Decompression (CD) is an important method for the treatment of OsteoNecrosis of the Femoral Head (ONFH). Avascular Necrosis (AVN) of the femoral head usually occurs in the third to fifth decade. We wanted to study the safety and effectiveness of core decompression and cancellous bone grafting for AVN of femoral head.

\section{METHODS}

Our study is a longitudinal observational study of 30 patients (26 males and 4 females) out of which 16 patients had unilateral involvement. Patients were clinically evaluated for pain, and presence of limping. During the follow up, they were clinically examined, and $\mathrm{x}$-ray was done to evaluate improvement or deterioration.

\section{RESULTS}

In our study, for all 30 patients, Harris Hip Score was used to assess the functional outcome of those patients and VAS score was used to assess the improvement in pain and excellent results were found from those patients who had anterosuperior quadrant lesion which was small in size and when the graft was given up to $5 \mathrm{~mm}$ subchondral bone.

\section{CONCLUSIONS}

Core decompression is an effective and safe method of treating ONFH. Core decompression with iliac crest bone grafting may be a treatment modality in the early stages of osteonecrosis of femoral head. (Stage I and II)

\section{KEY WORDS}

Avascular Necrosis Femoral Head, Core Decompression, Cancellous Bone Grafting
Corresponding Author: Dr. Debojyoti Mukherjee, 2/A PC Sarkar Sarani, Kolkata - 19, West Bengal, India.

E-mail: debojyoti64@gmail.com

DOI: $10.14260 /$ jemds/2020/845

How to Cite This Article:

Mukherjee D, Halder R. Core decompression and cancellous bone grafting for avascular necrosis of the femoral head - short term follow up. J Evolution Med Dent Sci 2020;9(51):3852-3855, DOI:

10.14260/jemds/2020/845

Submission 11-12-2019,

Peer Review 30-10-2020,

Acceptance 05-11-2020,

Published 21-12-2020.

Copyright (C) 2020 Debojyoti Mukherjee et al. This is an open access article distributed under Creative Commons Attribution License [Attribution 4.0 International (CC $B Y 4.0)]$ 


\section{BACKGROUND}

Osteonecrosis is a progressive disease of impaired osseous blood flow with wide ranging aetiology and commonly affecting patients in third, fourth and fifth decade of life. It can lead to collapse and joint destruction. ${ }^{1}$ Most of these are idiopathic and are young when diagnosed. Osteonecrosis is a disease arising from impaired osseous blood flow which can follow traumatic or non-traumatic conditions. Osteonecrosis of femoral head, a disabling condition is often seen in association with many disorders like corticosteroids consumption, haemoglobinopathy viz., sickle cell disease and coagulopathies, alcohol abuse, certain renal, hepatic and skin disorders and it commonly affects young patients around the age 20 - 40 years. Many factors with strong associations have been identified, including long term or high dose steroid use, moderate to heavy alcohol consumption, trauma, blood clotting disorders, and so on. There are various modalities of conservative and surgical methods to halt the natural history of the disease. Principle of core decompression is based on the belief that the procedure relieves intraosseous pressure caused by venous congestion, allowing improved vascularity and possibly slowing the progression of the disease. Success rates of core decompression can be further increased by structural bone grafting techniques after core decompression using cortical bone, cancellous bone, vascularized bone graft, and debridement of necrotic bone from the femoral head. Steinberg et al., 2 reported that central decompression attained only a $64 \%$ success rate, and an autologous bone graft is advised to be performed simultaneously. Core decompression procedures have been performed alone, or with various forms of vascularized bone grafting, electrical stimulation, and other adjuvant therapies. The rate of clinical success of these combined surgical procedures has ranged from $33 \%$ to $95 \%{ }^{3}$

Though there is a wide range of treatment modalities available but still the management of AVN of femoral head remains controversial. Medical treatment includes nonsteroidal anti-inflammatory drugs, bisphosphonates, hyperbaric oxygen therapy, and extracorporeal shock wave therapy, but their role has been shown to be ineffective whereas on the other hand, surgical options are femoral head preserving or hip salvaging procedures and hip replacement procedures. Preservation of femoral head delays the replacement procedures which have finite lifespan. Hence, femoral head-preserving procedures should be considered for the management of properly selected patients in order to delay the arthroplasty and also preservation of head allows for floor level activities which are required for Indian population. Therefore, we envisage to analyse the clinical outcome and radiological outcomes after core decompression and iliac crest bone grafting.

\section{METHODS}

It is a longitudinal observational study conducted among 30 patients who were selected and examined thoroughly. Proper past history was taken regarding any specific medications, addiction etc. At first instance they were examined clinically to evaluate pain or any limping. Range of motion mainly rotation (internal and external) and note the ambulatory distance at a stress without pain. All patients had done x-ray and frog leg lateral view and MRI (Magnetic Resonance Imaging). The patients with pre-collapse stages were selected. For the staging Ficat and Arlet classification was used, so up to stage 2 the patients were treated with core decompression with autogenous iliac crest bone graft. All the patients were followed up for 24 months. During the follow up they were clinically examined, and x-ray done to evaluate improvement or deterioration.

\section{Inclusion Criteria}

1. Non traumatic origin.

2. Both sex and age $<55$ yrs.

\section{Exclusion Criteria}

1. Ficat and Arlet stage III \& IV.

2. Osteonecrosis following trauma.

\section{Surgical Technique}

After thorough check up and preparation the patients were operated under spinal anaesthesia. Incision over iliac crest was given to take the bone graft and then prepared into small pieces and kept in a pot mixed with blood. The surgeries were done under spinal anaesthesia, in supine position on a fracture table and under image intensifier. The lateral femoral cortex was exposed through a small linear incision which was given over the lateral aspect of greater trochanter of femur and beginning the incision at the vastus ridge and carrying it out distally. Continuing dissection through the iliotibial band and splitting the fascia of the vastus lateralis muscle longitudinally. Elevating the vastus lateralis anteriorly off the lateral intermuscular septum while coagulating branches of the profound femoris artery as they are encountered. Completing the exposure by incising the origin of the vastus lateralis to allow the retraction and exposure of the trochanteric region. Following guide wire placement and reaming, curettage was done thoroughly. Then the prepared graft was applied and impacted. Same procedure was done in opposite side where pathology already had set in. In the post-operative period, compression bandage was applied for the ASIS graft site until removal of sutures. Active range of motion exercise of knee and ankle was started during the immediate postoperative period and were advised for bed rest for 2 months and then partial weight bearing was allowed. Full weight bearing with walker started after 3 months. All the patients were given alendronate post operatively.

All the data was entered into Microsoft excel. The results were expressed using descriptive statistics. Harris Hip Score was used to assess the functional outcome of those patients and VAS (Visual Analogue Scale) score was used to assess the improvement of the pain. All the patients were followed up at 6 months, 1 year and at 2 years clinically and radiologically (Xray and MRI at 24 months).

\section{RESULTS}

Majority of the study participants were in the age group of 36 - 45 years [Figure 1]. Most of them i.e., 26 / 30 i.e., $86.7 \%$ 
males and remaining 4 / 30 i.e., $13.3 \%$ were females. 16 patients i.e., $53.3 \%$ had unilateral involvement and the most common cause was idiopathic followed by steroid use and alcohol [Figure 2]. The successful outcome was noted among $86.3 \%$. We had 8 patients of stage I from which 6 had excellent results and rests 2 had good results. And from stage II we had 8 patients with excellent results, 3 patients had good results, 2 had fair results and 1 patient had poor outcome. So overall total 19 patients had excellent results $63.6 \%$ ) and 7 had good results $(22.7 \%)$ and 3 had fair results $(9.1 \%)$ and 1 had poor results (4.5\%). [Figure 3] The patients with poor results ultimately THR (Total Hip Replacement) was done in later stages. Excellent results were found from those patients who had antero-superior quadrant lesion and that was small sized, and the graft was given up to $5 \mathrm{~mm}$ subchondral bone. The patients with unilateral involvement had better outcome than bilateral cases. There was reduction in the average VAS scores in the post-operative period. [Figure 4]
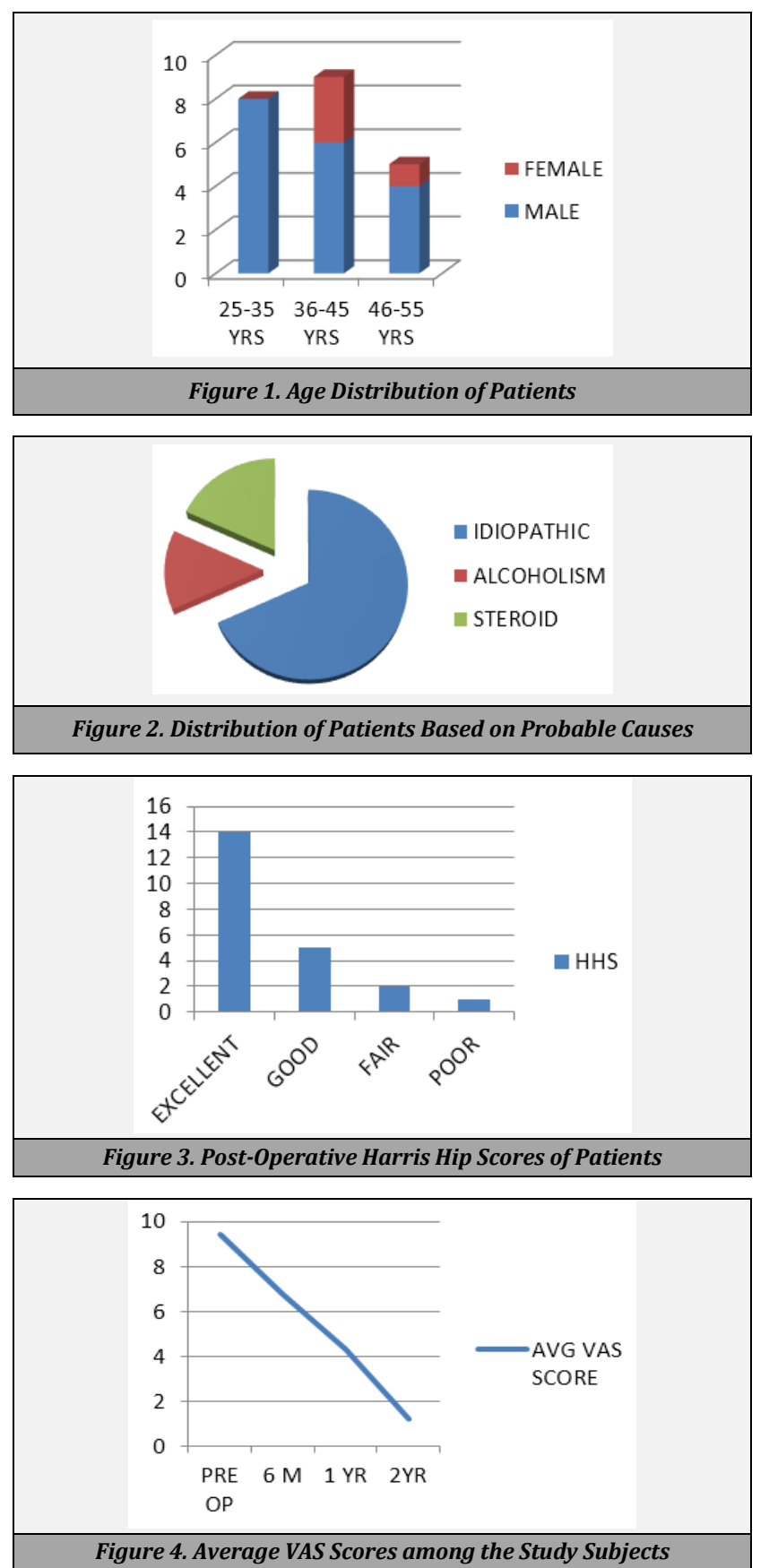

\section{DISCUSSION}

Treatment of ischemia of femoral head has become more common as many of the cases are detected in early stage. Early diagnosis before the appearance of radiological changes is very important of avascular necrosis. Diagnosis is based on clinical examination followed by investigations viz., $\mathrm{x}$-ray, bone scan, CT scan and MRI. However, x-ray has very limited significance in early stage of the disease. MRI helps in the early diagnosis of avascular necrosis when only bone marrow changes are noted.

Core decompression procedure provides a prospect to study histological changes of early bone ischemia and reduces the symptoms of pre collapse stage of ischemic necrosis because of reduction of pressure in compartment. There is no significant role of conservative management in osteoporosis of femoral head. The ideal treatment modality should be simple, reproducible and with low morbidity and mortality. The main advantages of non-vascularized graft are significant reduction in pain, early mobilisation, easy and technically less demanding procedure, less chance of donor side morbidity, less chance of iatrogenic sub-trochanteric fractures and shorter operative time.

\begin{tabular}{|ccc|}
\hline Series & Results & Follow Up \\
\hline Bucklety et al & $90 \%$ & 8 Yrs. \\
\hline Mont et al & $86 \%$ & 4 Yrs. \\
\hline Our Study & $86.3 \%$ & 2 Yrs. \\
\hline \multicolumn{2}{|c|}{ Table 1. Comparison of Our Study with Others } \\
\hline
\end{tabular}

Avascular necrosis of the femoral head is progression to collapse and eventual degenerative arthritis of the hip. The progression usually occurs within 12 to 24 months after diagnosis. The disease usually affects young people, so if possible, one should avoid or delay replacement arthroplasty.4-6 So, our goal is to preserve the femoral head. Various treatment modality is tried such as core decompression with and without vascularised and nonvascularised bone grafts, with structural and non-structural bone grafts. ${ }^{7-9}$ Roth et al $^{10}$ concluded that in early and potential reversible stages of non-traumatic osteonecrosis of femoral head or stage 1 and stage 2 according to Association Research Circulation Osseous (ARCO) and if the area of necrosis is medial or central and $<30 \%$, core decompression is indicated and should be offered. Sadileet al ${ }^{11}$ concluded that core decompression for ONFH are, at best, no better than other joint preserving strategies in terms of the efficacy and effectiveness. Patients with necrotic lesion size $<50 \%$ had the best outcomes with core decompression according to Rajagopalet al. ${ }^{12}$ Although core decompression is a standard treatment option to prevent THA (Total Hip Arthroplasty) in early stages of osteonecrosis, there are no rigorous studies that provide data regarding long-term health outcomes currently. Compared to external rotation, internal rotation showed more improvement in the study conducted by Shah et al. ${ }^{13}$ Though majority had pain relief immediately following operation among 28 hips, deterioration was seen as time passed. At 3 months, pain relief was noted in 22 hips but at final follow-up, only 19 hips had pain relief. Less than quarter of the hips required a replacement or salvage procedure. The success rates among the subjects with grades I, IIA and IIB were 92.3 
$\%, 100 \%$ and $50 \%$ respectively. The authors also concluded core decompression and bone grafting to provide satisfactory outcome when in early stages of the disease i.e., before the stage of collapse. In our study the excellent and good results from stage I is $75 \%$ and from stage II is $78.5 \%$. Total 22 patients had pain relief at final follow up our study and clinically internal rotation seems to be better achieved than external rotation. Less than $5 \%$ cases required replacement surgery. Ficat and Arlet reported good results in 80 to $90 \%$ of patients after core decompression alone.

The main purpose of our study was to make the patients pain free and delaying the need of arthroplasty at early age. The result of our study approximately matches with those studies' end results.

\section{CONCLUSIONS}

Osteonecrosis of femoral head is common in males in the third decade of life. It is very important to diagnose the disease as early as possible to avoid arthroplasty. At least for the first 2 months, weightbearing should be strictly avoided. Better outcome was found in small and medium sized lesions and where the necrotic areas are impacted with iliac crest bone graft after thorough curettage. So, core decompression with iliac crest bone grafting may be a treatment modality in early stages of osteonecrosis of femoral head (Stage I and II).

Data sharing statement provided by the authors is available with the full text of this article at jemds.com.

Financial or other competing interests: None.

Disclosure forms provided by the authors are available with the full text of this article at jemds.com.

\section{REFERENCES}

[1] Nixon JE. Avascular necrosis of bone: a review. J R Soc Med 1983;76(8):681-92.

[2] Steinberg ME, Larcom PG, Strafford B, et al. Core decompression with bone grafting for osteonecrosis of the femoral head. Clin Orthop Relat Res 2001;(386):71-8.

[3] Mont MA, Hungerford DS. Non-traumatic avascular necrosis of the femoral head. J Bone Joint Surg Am 1995;77(3):459-74.

[4] Bailey JP, Miller MI, eds. Osteonecrosis of the femoral head: treatment before collapse- a critical review. Adv Orthop Surg 1988;12:55.

[5] Aaron RK, Lennox D, Bunce GE, et al. The conservative treatment of osteonecrosis of the femoral head. A comparison of core decompression and pulsing electromagnetic fields. Clin Orthop Relat Res 1989;249:209-18.

[6] Bradway JK, Morrey BF. The natural history of the silent hip in bilateral atraumatic osteonecrosis. J Arthroplasty 1993;8(4):383-7.

[7] Brunelli G, Brunelli G. Free microvascular fibular transfer for idiopathic femoral head necrosis: long-term follow-up. J Reconstr Microsurg 1991;7(4):285-95.

[8] Bonfiglio M, Yoke E. Aseptic necrosis of the femoral head and non-union of the femoral neck. Effect of treatment by drilling and bone-grafting (Phemister technique). J Bone Joint Surg Am 1968;50(1):48-66.

[9] Boettcher WG, Bonfigilo M, Smith K. Non-traumatic necrosis of the femoral head. II. Experiences in treatment. J Bone Joint Surg Am 1970;52(2):322-9.

[10] Roth A, Beckmann J, Bohndorf K, et al. S3 - Guideline nontraumatic adult femoral head necrosis. Arch Orthop Trauma Surg 2016;136(2):165-74.

[11] Sadile F, Bernasconi A, Russo S, et al. Core decompression versus other joint preserving treatments for osteonecrosis of the femoral head: a meta analysis. $\mathrm{Br}$ Med Bull 2016;118(1):33-49.

[12] Rajagopal M, Samora JB, Ellis TJ, et al. Efficacy of core decompression as treatment for osteonecrosis of ther hip: a systemic review. Hip Int 2012;22(5):489-93.

[13] Shah SN, Kapoor CS, Jhaveri MR, et al. Analysis of outcome of avascular necrosis of femoral head treated by core decompression and bone grafting. J Clin Orthop Trauma 2015;6(3):160-6. 